\title{
HIV Pre-exposure Prophylaxis: Clinical Implications and Real-World Effectiveness
}

\author{
Julius Li ${ }^{1,2}$, Staci L. Dufrene ${ }^{1}$ and Jason F. Okulicz ${ }^{3 *}$ \\ ${ }^{1}$ South Texas Veterans Health Care System, Audie L. Murphy Hospital, San Antonio, TX, USA \\ ${ }^{2}$ University of Texas at Austin College of Pharmacy, Pharmacotherapy Education and Research Center, San Antonio, TX, USA \\ ${ }^{3}$ Infectious Disease Service, San Antonio Military Medical Center, San Antonio, TX, USA
}

\begin{abstract}
The use of tenofovir-emtricitabine as pre-exposure prophylaxis (PrEP) represents the latest strategy to prevent the acquisition of HIV by uninfected individuals. Despite clinical trials exhibiting significant risk reductions with PrEP, several limitations may hinder its real-world effectiveness. PrEP functions as one component of a comprehensive prevention program, which should also include intensive counseling on the importance of adherence, reduction of high-risk behaviors, and continued use of traditional preventative methods. Additionally, the greatest impact of PrEP, both clinically and financially, will likely arise from careful application in select high-risk populations. If properly implemented, PrEP has the potential to significantly reduce the incidence of new HIV infections.
\end{abstract}

\section{Introduction}

Since the discovery of the human immunodeficiency virus (HIV) in the 1980s, the AIDS epidemic has been responsible for the deaths of an estimated 30 million individuals [1]. In recent years, advances in the treatment of HIV have reduced overall AIDS-related mortality, but the continued transmission of HIV results in roughly 7,000 new infections daily. Current methods to prevent new infections, including the use of condoms, reduction of high-risk behaviors, and male circumcision, have remained relatively unchanged. Although these strategies have contributed to the stabilization or decline of new infections in many countries, the success of antiretroviral therapy (ART) has increased the global prevalence of HIV with more than 34 million individuals living with HIV worldwide, including approximately 1.2 million persons within the United States [2]. The improved survival of these individuals that now require lifelong therapy and care creates unsustainable economic and logistic burdens on healthcare systems, thus highlighting the need for novel preventative measures.

Past experiences in the prevention of infectious diseases throughout human history underscored the utility of vaccines as the optimal tool to prevent viral infections. With regards to vaccines for HIV, initial phase III trials were terminated early due to lack of efficacy. The more recent ALVAC-AIDSVAX B/E trial that utilized the prime-boost concept with a combination of vaccines demonstrated a modest relative risk reduction of approximately $30 \%$ [3]. Although this proof-of-concept study represents an important milestone in HIV vaccine research, response rates were far from optimal, and a viable candidate is likely several years away. Taking into additional consideration the time needed to manufacture and distribute these products, estimates project up to 60 million new infections before the widespread implementation of an effective vaccine [4]. In the interim, novel prevention strategies will likely involve the use of antiretroviral agents.

\section{Pre-Exposure Prophylaxis}

The use of antiretroviral agents to prevent the acquisition of HIV in uninfected individuals is primarily used in two scenariosperinatal and post-exposure prophylaxis. However, these strategies are constrained by their limited populations and narrow timeframe in which ART needs to be administered. More effective widespread use of antiretrovirals to prevent new HIV infections will require the use of ART prior to exposure. Although pre-exposure prophylaxis (PrEP) is currently used in other clinical situations, such as to prevent malaria in travelers to endemic regions, PrEP was not initially thought to be feasible for HIV due to significant toxicities and pill burdens of older antiretroviral agents.

The rationale for using PrEP to prevent HIV transmission is to limit the founder population of virus below a theoretical threshold needed to establish infection and to block viral replication to allow host immune responses to eliminate this smaller inoculum [4]. Although the use of multiple agents results in greater drug costs and risk of toxicities, single agent therapy increases the risk of inadvertent monotherapy. Because of the resultant increased risk of resistance, single agent therapy may also require more intensive HIV testing than has ever been achieved to date. Furthermore, preliminary animal studies with simian immunodeficiency virus (SIV) in macaques demonstrated superior benefits of combination therapy through more robust protection as well as decreased potential for the development of resistance [4]. Therefore, antiretroviral combinations were further pursued in human clinical studies.

Since PrEP involves long-term drug exposure in healthy asymptomatic individuals, antiretrovirals used for this indication require simple dosing schedules and favorable toxicity and interaction profiles. Additionally, ideal agents for PrEP should also exhibit adequate penetration into the rectal and genital mucosa, higher barriers to resistance, and activity before the integration of viral genetic material into the host genome. For these reasons, tenofovir disoproxil fumarate (TDF) and emtricitabine (FTC) emerged as viable candidates for PrEP and have been utilized in several clinical trials

*Corresponding author: Jason F. Okulicz, Infectious Disease Service, San Antonio Military Medical Center, San Antonio, Brooke Army Medical Center, 3551 Roger Brooke Drive, Fort Sam Houston, TX 78234-6200, USA, Tel: (210) 9164355; Fax: (210) 916-5900; E-mail: Jason.okulicz@amedd.army.mil

Received November 29, 2012; Accepted November 30, 2012; Published December 03, 2012

Citation: Li J, Dufrene SL, Okulicz JF (2012) HIV Pre-exposure Prophylaxis: Clinical Implications and Real-World Effectiveness. J AIDS Clinic Res 3:e110. doi:10.4172/2155-6113.1000e110

Copyright: (c) $2012 \mathrm{Li} \mathrm{J}$, et al. This is an open-access article distributed under the terms of the Creative Commons Attribution License, which permits unrestricted use, distribution, and reproduction in any medium, provided the original author and source are credited. 


\begin{tabular}{|c|c|c|c|c|c|c|}
\hline Study & Design & Location & Population & Intervention arms & $\operatorname{RR}(95 \% \mathrm{Cl})$ & ARR \\
\hline iPrEx & Phase III, safety and efficacy & $\begin{array}{l}\text { Brazil, Ecuador, Peru, South Africa, } \\
\text { Thailand, United States }\end{array}$ & 2499 MSM & Daily oral TDF-FTC & $44 \%(15,63)$ & $2.2 \%$ \\
\hline TDF2 & Phase III, safety and efficacy & Botswana & 1219 heterosexual men and women & Daily oral TDF-FTC & $75 \%(55,87)$ & $2.5 \%$ \\
\hline Partners PrEP & Phase III, safety and efficacy & Kenya, Uganda & $\begin{array}{l}4758 \text { serodiscordant heterosexual } \\
\text { couples }\end{array}$ & $\begin{array}{l}\text { Daily oral TDF; } \\
\text { Daily oral TDF-FTC }\end{array}$ & $62 \%(22,83)$ & $2.4 \%$ \\
\hline FEM-PrEP & $\begin{array}{l}\text { Phase III, safety and } \\
\text { effectiveness }\end{array}$ & Kenya, Tanzania, South Africa & 2120 heterosexual women & Daily oral TDF & $6 \%(-52,41)$ & $0.2 \%$ \\
\hline
\end{tabular}

RR: Relative Risk; ARR: Absolute Risk Reduction; MSM: Men Who have Sex with Men; TDF-FTC: Tenofovir-Emtricitabine; TDF: Tenofovir disoproxil fumarate Table 1: Clinical trial design and results from published PrEP studies.

(Table 1). Both agents have an extensive track record of safety in the past decade of use in HIV-infected individuals with few reports of serious toxicities. Moreover, two phase II safety studies revealed no short-term differences in the frequency of adverse events between subjects taking oral tenofovir and placebo in both men who have sex with men (MSM) in the United States and female sex workers in subSaharan Africa [5,6]. Nevertheless, longer-term exposures to TDF or TDF-FTC in HIV-infected populations increase the risk of side effects, such as the potential for kidney injury, decreased bone mineral density, and gastrointestinal effects.

Current topical preparations of tenofovir in development achieve much higher rectal and genital tissue concentrations while minimizing the potential for systemic toxicities. However, the hyperosmolarity of these formulations may increase susceptibility to HIV by disrupting genital and rectal epithelium [7]. Due to stability issues, these microbicides are not formulated with the disoproxil prodrug of tenofovir, which has demonstrated better tissue penetration and more potent antiviral activity compared with tenofovir alone [7]. These factors may help to partially explain the conflicting clinical evidence regarding topical formulations of tenofovir. Results from the CAPRISA-004 study demonstrated a $39 \%$ relative reduction in the incidence of HIV with topical tenofovir, but the topical arms of the VOICE MTN-003 were discontinued due to futility $[8,9]$. Given these divergent results and the lack of licensed topical antiretrovirals, clinical studies examining the effects of PrEP focused on oral TDF-FTC.

\section{Clinical Efficacy and Safety}

The safety and efficacy of TDF-FTC for HIV PrEP was evaluated in four randomized, controlled trials. These trials included young healthy subjects from broad patient populations at high-risk of acquiring HIV infection. All four studies stipulated subjects' participation in comprehensive prevention programs as adjunct interventions to PrEP, which included counseling on the importance of adherence, reduction of high-risk behaviors, and continuation of traditional prevention methods. The subjects in these studies represented individuals at substantial risk for acquiring HIV. Roughly $80 \%$ of the MSM in the iPrEx study practiced unprotected receptive anal intercourse with an average of 18 sexual partners over a span of only 12 weeks [10]. The HIV-negative subjects of serodiscordant couples in Partners-PrEP were consistently engaging in intercourse with their untreated HIVpositive partners [11]. Both TDF2 and FEM-PrEP were conducted in sub-Saharan Africa where the prevalence of HIV is greater than $20 \%$ in some countries $[12,13]$.

Significant risk reductions were demonstrated in three of these studies, specifically in high-risk MSM, serodiscordant couples, and high-risk heterosexuals [10-13]. However, the FEM-PrEP study was terminated due to futility, but this lack of efficacy was primarily attributed to high rates of nonadherence. Self-reported rates of adherence exceeded $90 \%$, but less than $40 \%$ of subjects had detectable drug levels [13]. The low level of adherence was likely due to the majority of individuals perceiving themselves to be a little to no risk of acquiring HIV. Interestingly, relatively high rates of pregnancy were observed in the female subjects taking oral contraceptives possibly indicating difficulty maintaining daily pill regimens.

Although the absolute risk reductions of 2-3\% appear unimpressive, these reductions are potentially compounded by downstream effects particularly since the sexual partners of the MSM in iPrEx presumably also had an average of 18 sexual partners. Similarly, the benefits of PrEP are likely amplified in serodiscordant couples in which the HIVpositive individual remains untreated due to their partner's lifetime HIV exposure. On the other hand, the results of TDF2 are less likely to be applicable to countries with low HIV prevalence, such as the United States. Given the prevalence rates of greater than $10 \%$ in many subSaharan countries, the probability of encountering an HIV-infected individual is 1 in 5-10 persons as compared to 1 in 100 compared to countries with lower prevalence rates. Therefore, although the results from trials are promising, they need to be considered in the context of not only the subjects' baseline characteristics but also the environment in which they were studied.

TDF-FTC has a strong track record of safety for almost a decade in HIV-infected individuals, and these four landmark trials demonstrated a similar safety profile in uninfected persons. No differences in serious adverse events or laboratory abnormalities defined as Grade 3 or higher were observed in subjects receiving TDF-FTC for PrEP across all four trials [10-13]. Although bone mineral density scores were significantly lower in patients receiving TDF-FTC in the TDF2 study, these differences were small and of unknown clinical significance [12]. Discontinuation rates due to adverse events were also similar to placebo across all trials with complete resolution of toxicities once the study drugs were stopped. Thus, TDF-FTC has similar safety profiles in both HIV-infected and uninfected persons at least in the short-term, but longer-term safety data with drug exposures greater than 2 years are needed.

\section{Effectiveness of Pre-Exposure Prophylaxis in Real- World Settings}

The divergent results from these studies raise concerns about the real-world effectiveness of PrEP. Although the primary driver for futility in the FEM-PrEP trial was non-adherence, other potential limitations of PrEP include risk compensation and the development of resistance.

\section{Adherence}

Currently, we lack standardized, objective measures of adherence forcing us to rely on self-reporting and its inherent problems as seen in the FEM-PrEP study. Self-reported rates in the FEM-PrEP study were about $95 \%$, but drug testing revealed detectable levels in less than $40 \%$ of subjects sampled [14]. Throughout the published PrEP trials, 
high levels of adherence were also associated with greater efficacy. In the iPrEx study, increased risk reduction was seen at higher rates of adherence. Specifically, pill use greater than $50 \%$ and $90 \%$ resulted in relative risk reductions of $50 \%$ and $73 \%$, respectively [10]. Furthermore, detectable drug levels were indicative of recent pill use and thus, adherence and efficacy. Seroconverters had lower rates and levels of detectable drug concentrations compared to nonseroconverters across all four trials. Moreover, both iPrEx and Partners-PrEP demonstrated a risk-reduction of more than $90 \%$ associated with detectable drug levels.

Similar to HIV-infected individuals, clinicians need to identify barriers to adherence in uninfected individuals to optimize the effectiveness of PrEP. Addressing these barriers is particularly important in this patient population since the majority of women in the FEM-PrEP study perceived themselves to be at little to no risk for HIV infection. Additionally, qualitative studies have demonstrated that persons most willing to use PrEP also have the lowest self-perceived risk and may not be as conscientious about strict adherence to daily TDF-FTC $[15,16]$. In serodiscordant couples, trust and commitment between partners will also likely play an important role in levels of adherence.

\section{Risk compensation}

Risk compensation has the potential to mitigate the benefits of any preventative measure through increases in risk-taking behavior. With regards to PrEP, potential users may perceive themselves to be better protected against HIV, and thus, become more lax about consistent condom use and other high-risk sexual behaviors. Evidence from non-PrEP settings has shown varying amounts of risk compensation that depended on the degree of perceived benefits as well as associated toxicities and cost of therapy. Although HIV-positive patients receiving antiretroviral therapy (ART) did not exhibit increased high-risk sexual behaviors, subjects with any HIV status that believed ART and undetectable viral load protected against HIV transmission were more likely to engage in unprotected sex [17]. On the other hand, a longitudinal cohort study of patients receiving an advanced supply of antiretrovirals for post-exposure prophylaxis demonstrated no increases in risk behavior due to uncertain protection from HIV infection [18]. Therefore, risk compensation varies between different modalities based on their perceived benefits as well as adverse events and financial cost.

Recent media reports of the underground use of tenofovir by MSM at dance clubs in a practice commonly referred to as "taking a T" or as part of a cocktail known as the 3 V's (Viread, Valium, and Viagra) raises concern about potential abuse and misuse of TDF-FTC $[19,20]$. In context of clinical trials, there was no evidence of increase in risky behaviors and actually increases in safer sex practices in some studies. However, these patients received PrEP in conjunction with intensive monthly counseling on risk-reduction behaviors. As mentioned earlier, qualitative studies evaluating the potential for risk compensation in PrEP candidates revealed significant differences between their actual and perceived risks for acquiring HIV. Taken together, these findings further underscore the role of PrEP as one component of a comprehensive prevention program.

\section{Resistance}

The primary resistance pathways for FTC and TDF involve the mutations $\mathrm{M} 184 \mathrm{~V}$ and $\mathrm{K} 65 \mathrm{R}$, respectively. FTC induces the M184V resistance mutations relatively rapidly within 15 days of monotherapy, but it also confers reduced replicative capacity of the mutated virus. Furthermore, the development of M184V during combination therapy with TDF-FTC has been shown to delay the emergence of K65R [21,22]. Since both TDF and FTC are components of preferred antiretroviral combinations for the treatment of HIV, the development of resistance in patients failing PrEP could have important implications for the treatment of HIV in these individuals. Resistance to first-line combinations limit treatment options in PrEP failures to alternative regimens with significantly more toxicities and greater pill burdens potentially compromising adherence and efficacy.

Current clinical trial evidence revealed no resistance mutations detected in patients that became HIV-positive after enrollment, but this may be a byproduct of low adherence levels in these subjects. Since seroconverters were less likely to have detectable drug levels, their infecting HIV strain had minimal drug exposure thereby decreasing the potential for resistance in these individuals. Of greater concern is the development of resistance in HIV-infected patients inadvertently started on PrEP. Resistance mutations may emerge in individuals with undetected HIV-1 infections because FTC-TDF only does not comprise a complete treatment regimen for HIV-infected individuals. These mutations were evident in all six subjects who were found to be HIV-positive at baseline and initiated on TDF-FTC. The development of resistance in these subjects illustrates the importance on establishing seronegativity in potential PrEP candidates.

\section{Cost-effectiveness}

Since the combination of TDF-FTC is currently available as branded Truvada ${ }^{\circ}$, widespread implementation of PrEP will require significant financial investments. The average monthly cost of oncedaily TDF-FTC ranges from $\$ 700-\$ 900$ in the United States. Three analyses to date have been conducted to evaluate the cost-effectiveness of implementing PrEP in MSM populations [23-25]. Conservative estimates of PrEP use in all MSM resulted in a cost of $\$ 298,000$ per quality-adjusted life years (QALYs) gained with the most optimistic predictions of approximately $\$ 30,000$ per QALY gained. Across all analyses, lower cost per QALY and potentially even cost-savings were seen if PrEP was only implemented in the highest risk patients (e.g. an average of 5 annual partners). Currently, the average American appears willing to accept a cost of a little over $\$ 100,000$ per QALY gained for most general health interventions [26]. Although Gilead Sciences, the manufacturer of both drugs, is working with the World Health Organization (WHO) to provide TDF-FTC at discounted prices of $\$ 26$ per month to developing countries, implementation of PrEP will still require significant costs due to training of healthcare workers for adequate screening, monitoring, and counseling [27]. Therefore, the cost-effectiveness of PrEP may ultimately vary depending on the drug costs as well as degree and setting of implementation.

\section{The End of AIDS?}

PrEP with TDF-FTC represents the newest biomedical intervention to help turn the tide of the AIDS epidemic, but it should not be considered the magic bullet to prevent HIV transmission. The success of PrEP will be limited by levels of adherence, degree of risk compensation, and emergence of resistance as well as resource and financial limitations. PrEP should be optimally reserved for individuals with ongoing high-risk exposure to HIV, such as serodiscordant couples in which the HIV-positive partners are either unwilling or ineligible to start ART or high risk MSM. In terms of implementation in high-risk heterosexuals, PrEP will likely have its greatest impact in persons living in HIV endemic regions. PrEP should be initiated in conjunction with other preventative measures, including the proper 
Citation: Li J, Dufrene SL, Okulicz JF (2012) HIV Pre-exposure Prophylaxis: Clinical Implications and Real-World Effectiveness. J AIDS Clinic Res 3:e110. doi:10.4172/2155-6113.1000e110

Page 4 of 4

use of condoms, counseling on the reduction of high-risk behaviors, and the importance of strict adherence, regardless of the population in which it is implemented. If used appropriately, PrEP has the potential to further accelerate the decline in the incidence of new HIV infections, particularly in the absence of an effective vaccine on the horizon. Ongoing clinical studies and demonstration projects with anticipated completion dates within the next two years will hopefully address some of the concerns regarding the real-world effectiveness of PrEP.

\section{References}

1. World Health Organization, UNAIDS, UNICEF (2011) Global HIV/AIDS response: epidemic update and health sector progress towards universal access. Geneva, Switzerland.

2. UNAIDS (2010) Getting to Zero: 2011-2015 Strategy: Joint United Nations Programme on HIVIAIDS. Geneva, Switzerland.

3. Rerks-Ngarm S, Pitisuttithum P, Nitayaphan S, Kaewkungwal J, Chiu J, et al. (2009) Vaccination with ALVAC and AIDSVAX to prevent HIV-1 infection in Thailand. N Engl J Med 361: 2209-2220.

4. Kelesidis T, Landovitz RJ (2011) Preexposure prophylaxis for HIV prevention Curr HIVIAIDS Rep 8: 94-103.

5. Peterson L, Taylor D, Roddy R, Belai G, Phillips P, et al. (2007) Tenofovir disoproxil fumarate for prevention of HIV infection in women: a phase 2, doubleblind, randomized, placebo-controlled trial. PLoS Clin Trials 2: e27.

6. Centers for Disease Control and Prevention. Preliminary results from first safety study of daily tenofovir for HIV prevention among MSM find no significant concerns. Atlanta, Georgia.

7. Anderson PL, Kiser JJ, Gardner EM, Rower JE, Meditz A, et al. (2011) Pharmacological considerations for tenofovir and emtricitabine to prevent HIV infection. J Antimicrob Chemother 66: 240-250.

8. Abdool Karim Q, Abdool Karim SS, Frohlich JA, Grobler AC, Baxter C, et al (2010) Effectiveness and safety of tenofovir gel, an antiretroviral microbicide, for the prevention of HIV infection in women. Science 329: 1168-1174.

9. Microbicide Trials Network. Questions and Answers: VOICE: vaginal and oral interventions to control the epidemic. Pittsburgh, Pennsylvania.

10. Grant RM, Lama JR, Anderson PL, McMahan V, Liu AY, et al. (2010) Preexposure chemoprophylaxis for HIV prevention in men who have sex with men. N Engl J Med 363: 2587-2599.

11. Baeten JM, Donnell D, Ndase P, Mugo NR, Campbell JD, et al. (2012) Antiretroviral prophylaxis for HIV prevention in heterosexual men and women. N Engl J Med 367: 399-410.

12. Thigpen MC, Kebaabetswe PM, Paxton LA, Smith DK, Rose CE, et al. (2012)
Antiretroviral preexposure prophylaxis for heterosexual HIV transmission in Botswana. N Engl J Med 367: 423-434.

13. Van Damme L, Corneli A, Ahmed K, Agot K, Lombaard J, et al. (2012) Preexposure prophylaxis for HIV infection among African women. N Engl J Med 367: 411-422.

14. Crepaz N, Hart TA, Marks G (2004) Highly active antiretroviral therapy and sexual risk behavior: a meta-analytic review. JAMA 292: 224-236.

15. Golub SA, Kowalczyk W, Weinberger CL, Parsons JT (2010) Preexposure prophylaxis and predicted condom use among high-risk men who have sex with men. J Acquir Immune Defic Syndr 54: 548-555.

16. Whiteside YO, Harris T, Scanlon C, Clarkson S, Duffus W (2011) Self-perceived risk of HIV infection and attitudes about preexposure prophylaxis among sexually transmitted disease clinic attendees in South Carolina. AIDS Patient Care STDS 25: 365-370.

17. Crepaz N, Hart TA, Marks G (2004) Highly active antiretroviral therapy and sexual risk behavior: a meta-analytic review. JAMA 292: 224-236.

18. Roland ME, Neilands TB, Krone MR, Katz MH, Franses K, et al. (2005) Seroconversion following nonoccupational postexposure prophylaxis against HIV. Clin Infect Dis 41: 1507-1513.

19. Costello D (2005) AIDS pill as party drug? The Los Angeles Times.

20. Cohen J (2006) Protect or disinhibit? New York Times.

21. Hurt CB, Eron JJ Jr, Cohen MS (2011) Pre-exposure prophylaxis and antiretroviral resistance: HIV prevention at a cost? Clin Infect Dis 53: 12651270.

22. van de Vijver DA, Boucher CA (2010) The risk of HIV drug resistance following implementation of pre-exposure prophylaxis. Curr Opin Infect Dis 23: 621-627.

23. Desai K, Sansom SL, Ackers ML, Stewart SR, Hall HI, et al. (2008) Modeling the impact of HIV chemoprophylaxis strategies among men who have sex with men in the United States: HIV infections prevented and cost-effectiveness. AIDS 22: 1829-1839

24. Paltiel AD, Freedberg KA, Scott CA, Schackman BR, Losina E, et al. (2009) HIV preexposure prophylaxis in the United States: impact on lifetime infection risk, clinical outcomes, and cost-effectiveness. Clin Infect Dis 48: 806-815.

25. Juusola JL, Brandeau ML, Owens DK, Bendavid E (2012) The cost-effectiveness of preexposure prophylaxis for HIV prevention in the United States in men who have sex with men. Ann Intern Med 156: 541-550.

26. Braithwaite RS, Meltzer DO, King JT Jr, Leslie D, Roberts MS (2008) What does the value of modern medicine say about the $\$ 50,000$ per quality-adjusted life-year decision rule? Med Care 46: 349-356.

27. Paxton LA, Hope T, Jaffe HW (2007) Pre-exposure prophylaxis for HIV infection: what if it works? Lancet 370: 89-93. 